\title{
DÉTERMINISME DE LA CONSTRUCTION DES CELLULES DE MALES ET DES CELLULES D'OUVRIÈRES CHEZ APIS MELLIFICA
}

\author{
PAR \\ Roger DARCHEN \\ Station de Recherches Apicoles \\ Bure-sur-Yvette \\ et \\ Maurice VUILLAUME \\ Laboratoire de Zoologie \\ Faculté des Sciences Rennes
}

I.e problème du déterminisme de construction des cellules mâles et des cellules d'ouvrières chez Apis Mellifica n'a pas été étudié jusqu'à présent par beaucoup d'auteurs. I e présent travail met en évidence une influence possible du couvain àgé.

Dans une première série d'expériences, on introduit $\mathrm{I}, 5 \mathrm{~kg}$ d'abeilles de tout âge, de race noire commune, dans des ruches de $12 \mathrm{~cm} \times 45 \mathrm{~cm} \times 30 \mathrm{~cm}$. On ajoute 111 ou det1x cadres de couvain âgé en train de naître; chaque cadre est remplacé dès qu'il vient à s'épuiser. Il n'y a point de reine; on veille à nourrir abondamment au sirop de sucre; or, si on introduit une barrette de bois portant quelques taches de cire, les abeilles les étirent et en font de longues bâtisses constituées de cellutes de mâles. Si par hasard apparaissent des cellules d'ouvrières, nous en concluons d'habitude à l'introduction accidentelle d'une reine au sein du groupe et nous l'avons retrouvée dans tous les cas.

Une deuxième série d'expériences effectuées dans les mêmes conditions, saut qu'on n'introduit pas de courain naissant, permet de constater les phénomènes suivants :

\begin{tabular}{|c|c|c|c|}
\hline \multirow{2}{*}{ fours depuis la mise en ruche } & \multirow{2}{*}{$\begin{array}{l}\text { Vombre de } \\
\text { (nI }^{2} \text { const ruits }\end{array}$} & \multicolumn{2}{|c|}{ Types de cellules } \\
\hline & & o & $q$ \\
\hline $1 \ldots$ & 160 & $100 \%$ & $0 \%$ \\
\hline $2 \ldots$ & 110 & $100 \%$ & $0 \%$ \\
\hline$\therefore \ldots \ldots \ldots$ & $8, y$ & o " " & $100 \%$ \\
\hline $\begin{array}{c}t+1 \\
t\end{array}$ & -4 & $0 "$. & $100 \%$ \\
\hline 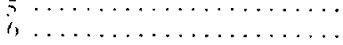 & $\begin{array}{l}+4 \\
28\end{array}$ & $\begin{array}{lll}0 & \ldots \\
0 & 0\end{array}$ & $\begin{array}{l}100 \% \\
100 \%\end{array}$ \\
\hline
\end{tabular}

Ise premier et le deuxième jour, les constructions spontanées se composent exclusivement de cellules de mâles et les jours suivants de cellules d'ouvrières. Ajoutons que du premier au sixième jour, la diminution de la surface construite est considérable et à peu près régulière. 
Dans une troisième série d'expériences, on divise une ruche populeuse en deux parties à peu près égales et l'on nourrit abondamment au sirop de sucre ; des deux populations ainsi créées, l'une est orpheline et l'autre possède une reine. Le tableau II résume les observations effectuées dans la partie orpheline : construction exclusive de cellules mâles les trois premiers jours, remplacées plus ou moins complètement par des cellules d'ouvrières les jours suivants. I a surface des édifices construits dans la partie qui possède une reine est + à 5 fois supérieure à celle des édifices de la ruche orpheline.

TABLEAT II

\begin{tabular}{|c|c|c|c|}
\hline \multirow[t]{2}{*}{ Jours } & \multirow{2}{*}{$\begin{array}{l}\text { Nombre de } \\
\text { cm }^{2} \text { construits }\end{array}$} & \multicolumn{2}{|c|}{ Types de cellules } \\
\hline & & $0^{*}$ & 7 \\
\hline I $\ldots \ldots \ldots \ldots \ldots \ldots$ & $\circ$ & & \\
\hline $2 \ldots \ldots \ldots \ldots \ldots \ldots$ & 120 & $100 \%$ & $0 \%$ \\
\hline $3 \ldots \ldots \ldots \ldots \ldots \ldots$ & 30 & $100 \%$ & $0 " \%$ \\
\hline $4 \ldots \ldots \ldots \ldots \ldots \ldots$ & 30 & $50 \%$ & $50 \%$ \\
\hline $\bar{\gamma} \ldots \ldots \ldots \ldots \ldots \ldots \ldots$ & 20 & $50 \%$ & $=0 \%$ \\
\hline $6 \ldots \ldots$ & 20) & $100 \%$ & $0 \%$ \\
\hline $7 \ldots \ldots \ldots \ldots \ldots \ldots$ & 30 & $5 \%$ & $15 \%$ \\
\hline $8 \ldots \ldots \ldots \ldots \ldots \ldots$ & $\geq 1$ & $0 \%$ & $100 \%$ \\
\hline $9 \ldots \ldots \ldots \ldots \ldots \ldots$ & Io & $0 \%$ & $100 \%$ \\
\hline $10 \ldots \ldots \ldots \ldots \ldots \ldots \ldots$ & 5 & $0 \%$ & $0 \%$ \\
\hline II $\ldots \ldots \ldots \ldots \ldots \ldots \ldots$ & 5 & $0 \%$ & 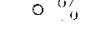 \\
\hline
\end{tabular}

\section{Ibiscussion.}

I'un de nous (DARCHEx, I956) a signalé la grande influence de la reine sur les constructions cirières; les petits groupes d'abeilles déponrvues de reine, ne construisent pas ; et pour pouvoir entreprendre la construction, le groupe d'abeilles orphelines doit comporter plusieurs milliers d'ouvrières. Ajoutons qu'une population d'abeilles en présence d'une vieille reine bourdonneuse peut transformer les cellules d'ouvrières en cellules de mâles. En revanche ces mêmes abeilles seront capables de transformer les cellules de mâles en cellules d'ouvrières si 1'on introduit une reine jeune et féconde; la reine induit la construction des cellules d'ouvrières; les cellules de mâles n'apparaissent que lorsque la population s'est beaucoup accrue et que peut-être la reine est incapable de fournir suffisamment d'ectohomone à une population trop nombreuse. BUTLER suppose aussi que cette carence d'ectohormone est la cause de la construction de cellules royales qui apparaissent alors. Nos expériences nous amènent à compliquer quelque peu les conclusions de Butrer. Il semble quiun second facteur favorisant la construction des cellules de mâles se trouve dans les abeilles naissantes ou très jeunes; l'action du couvain naissant peut alors affecter une double forme :

Io Elle peut être hormonale c'est-à-dire que les jeunes abeilles 
sécréteraient une substance plus ou moins antagoniste de l'ectohormone royale et favorisant la construction des cellules de mâles. Dans ce cas, il suffirait sans doute d'un nombre assez limité d'abeilles naissantes pour induire le phénomène.

$2^{\circ}$ I'action serait de nature trophique; on sait que la composition chimique et la teneur en vitamines des jeunes ouvrières est tout à fait différente de celle des ouvrières plus âgées. I,es modifications dans les échanges continuels de nourriture (dont Nixon et RibBaxids ont montré l'importance) seraient alors à la base des changements de direction de l'instinct constructeur. Il est plus difficile d'expliquer ce qui se passe dans la population d'abeilles en présence d'une vieille reine : ici en l'absence de couvain abondant, les abeilles construiraient néanmoins des cellules de mâles; toutefois la présence d'œufs non fécondés doit beaucoup modifier le phénomène, ainsi sans doute que la baisse de la sécrétion de l'ectohormone chez la vieille reine.

Il ne s'agit évidemment encore que d'hypothèses de travail que nous pensons vérifier ultérieurement.

\section{Résumé.}

I $^{\mathrm{L}} \mathrm{L}$ a reine inhibe incomplètement la construction des cellules de mâles dans les conditions spéciales de nos expériences.

$2^{\circ}$ I a présence de jeune couvain naissant inhibe la construction des cellules d'ouvrières.

\section{REFERENCES BIBI,IOGRAPHIQUES}

Butr.k:R (C. G.). - - Some further observations on the nature of " (queen substance " and its role on the organization of a honeybee community. Proc. Roy. Entom. Soc., A, 3I, I956.

I) ARChen (R.). - La reine d'Apis mollifica et les constructions cirières. $C$. R. Acad. Sc., 439-4I, I956.

Nixon (H. I.), RiBbands (C. R.). - Food transmission in the honeybee community. Proc. Roy. Soc., B, I40, 43, I952. 